\title{
VR-Based Virtual Test Technology and Its Application in Instrument Development
}

\author{
Tiantai Guo ${ }^{1}$ and Xiaojun Zhou ${ }^{2}$ \\ ${ }^{1}$ China Jiliang University, Hangzhou 310018, P.R. China \\ guotiantai@163.com \\ ${ }^{2}$ Zhejiang University, Hangzhou 310009, P.R. China \\ sky@zju.edu.cn
}

\begin{abstract}
Test plays a very important role in manufacturing, but the general practice so far cannot fully meet the demands of flexibility and efficiency required by a fast-changing market. To supply a new and flexible approach to the solution of various complex engineering testing problems, the concept of "VRbased virtual test technology" was put forward in the authors' previous works, which can be regarded as the combination of these three technologies: virtual reality (VR), test technology and computer simulation. This paper is the deepening of the authors' previous works. After elucidating the theoretical model of VR-based virtual test technology, its application in instrument development is discussed, and finally a prototype VR-based instrument development system is given to show the initial effects of the approach. The conclusion is that VRbased virtual test technology can play a very important and active role in testing and instrument development.
\end{abstract}

Keywords: Virtual reality (VR), test technology, computer simulation, instrument development, virtual test technology.

\section{Introduction}

Test plays a very important role in manufacturing, but the general practice so far can not fully meet the demands of flexibility and efficiency required by a fast-changing market. For example, in many cases tests can only be made with equipments with limited functions, detection of machine parts' faults and failures is often not timely, and test of nanometer-scale or large-scaled machineries and robots working in environments inconvenient for human beings has caused much concern.

Virtual reality (VR) offers a new solution to these problems. VR is a technology which has found successful application in many fields like medical training, education, computer games, molecule simulation, robot control, machine tool simulation, flight, ship and vehicle simulators, etc. [1]-[4] VR is a computer system used to create an artificial world in which the user has the impression of being in that world and with the ability to navigate through the world and manipulate objects in the world.

After exploring the requirements and features of VR, test technology and computer simulation, the authors put forward the concept of "VR-based virtual test technology" in previous research works, which can be regarded as the combination of these three 
technologies: VR, test technology and computer simulation, where VR supplies the interface and environment for test activities to take place, test technology shows the immediate aim and use of the approach, while computer simulation supplies the necessary algorithms for the solution of engineering test tasks.

To make VR-based virtual test technology practical in engineering, both theoretical research and prototype development are necessary. On the basis of the authors' previous researches, this paper put forward a modified theoretical model of VR-based virtual test technology. Furthermore, this paper gives a prototype application of VRbased virtual test technology in instrument development to show the initial effect of VR-based virtual test technology. The conclusion is that VR-based virtual test technology can play a very important and active role in testing and instrument development, with the rapid progress of computer technology and the coming up of more affordable and practical VR peripherals.

\section{VR-Based Virtual Test Technology}

\subsection{VR-Based Virtual Test Technology (VRVTT)}

"VR-based virtual test technology" is a concept put forward by the authors in previous works as an attempt to supply a new approach to the solving of difficult engineering test problems such as the testing of mechanisms of nanometer scale, or equipments working in areas difficult to access by traditional ways, and the basic idea is first to generate a virtual test environment with computer software supported by appropriate computer hardware and VR peripherals in accordance with specific applications, in which virtual reality (VR) serves not only as an interface but also as an interaction tool between the user and the virtual test environment, and then the test processes are interactively planned, optimized and simulated in a virtual test environment, while intermediate and final test results are visualized, analyzed and replayed in dynamic 3D forms. [5] [6].

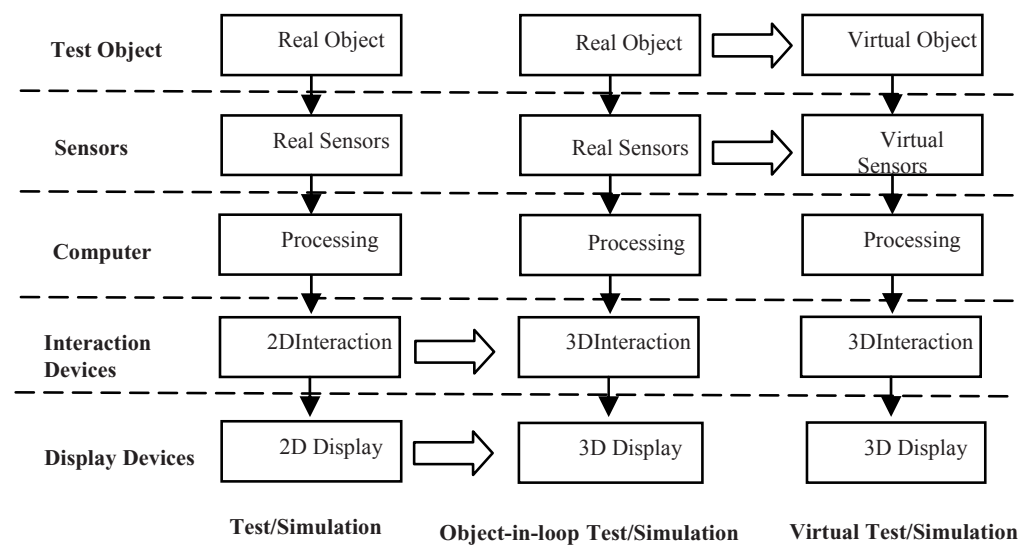

Fig. 1. Progress of test and simulation 
Fig.1 shows the progress of test and simulation, which shows a definite trend of substituting existing 2D interaction modes with 3D interaction devices such as spaceballs and trackers, and $2 \mathrm{D}$ display is giving way to $3 \mathrm{D}$ displays. This is what VR is expected to be doing so far. Furthermore, by substituting real objects with virtual (imaginary) objects, and real sensors with virtual sensors, we get VR-based virtual test technology (abbreviated as VRVTT henceforth), which can be used to simulate available test system for tutorial and training purposes, or to design new test equipments to show the principles and configurations of the equipments under design, or eventually to perform virtual tests completely in virtual test environment on the basis of theoretical modeling and simulation. Accordingly, the virtual test environment can either be a replica of actual world scenes, or any imagined or designed environment generated by computer.

VRVTT is a combination of these technologies: virtual reality (VR), computer simulation, test technology. VR supplies a platform for all the design, simulation and test activities, and computer simulation supplies the system with algorithms to make full use of the obtained data (real or virtual), while test is the direct purposes of the technology.

Depending on the function and whether real (physical) test object exists, the implementation of VRVTT drops under four categories (as shown in Table 1): physical test, object-in-loop test simulation, VR-based test and simulation, and complete VRbased test. The first two categories are the traditional way of conducting test and simulation, while the latter two are the trend of test and simulation technologies.

Table 1. Categories of VR-based virtual test technology

\begin{tabular}{|l|l|l|}
\hline & With real test object & Without real test object \\
\hline Test & Physical test & Complete VR-based test \\
\hline Simulation & Object-in-loop simulation & $\begin{array}{l}\text { VR-based test and simula- } \\
\text { tion }\end{array}$ \\
\hline
\end{tabular}

\subsection{Modified Theoretical Model of VRVTT}

The modified theoretical model of VRVTT is shown in Fig.2. This model expands the three-layered model of the original theoretical model of VRVTT into five interdependent layers, thus making the model more complete and practical. So now VRVTT can be classified into five layers:

1) Perception layer: this is the layer which supplies the user with a working virtual environment, and the user can interact directly with the virtual environment and check the process and results. "3Is" (Interaction, Immersion and Imagination) are the features of VR suggested by G. Burdea and P. Coiffet in Electro'93 International Conference[7], and their role is emphasized here to show the leading role of VR in VRVTT.

2) Function layer: this layer consists of the basic functions of VRVTT, including measurement, detection, test, simulation and training. 


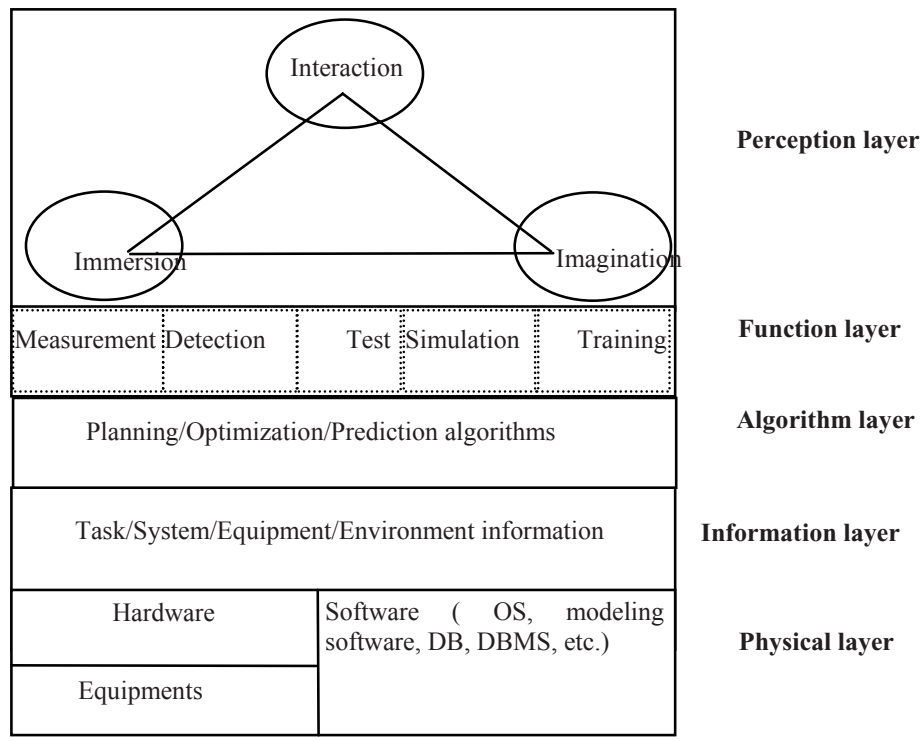

Fig. 2. Modified theoretical model of VRVTT

3) Algorithm layer: this layer integrates the algorithms necessary for the operation of VRVTT, such as planning algorithm, optimization algorithm, prediction algorithm, signal processing algorithms, etc.

4) Information layer: this layer includes the information of task description, measurement, detection and test system, equipment, environment, sensors, etc.

5) Physical layer: this layer is the physical basis of VRVTT, and it's made up of three parts:

a. Hardware, such as computer, peripherals, head-mounted display (HMD), etc.;

b. Equipments, which are a part of VRVTT when hardware-in-loop measurement, detection and test is performed;

c. Software, such as operating system (OS), modeling software, database (DB), database management system (DBMS), etc.

\subsection{Mathematical Model of VRVTT}

Theoretically speaking, VRVTT can be regarded as a reproduction of a real (physical) measurement, detection and test system, or merely an imaginary (virtual) visualization of a test system under design or simply in planning. To be of practical value, VRVTT must be similar to its physical or anticipated counterpart in features (geometrical, kinetic, dynamic, etc.), functions and working principles. Mathematically, the test process can be described as:

$$
\mathrm{T}=\{\mathrm{q} 0, \mathrm{c}, \delta, \mathrm{qf}\}
$$

Where $\mathrm{T}$ is the test process, while $\mathrm{q} 0$ is the initial (starting) conditions, $\mathrm{c}$ is the constraints, $\delta$ is the operations taken, and qf is the final conditions. Formula (1) shows 
that the test process is a process in which the system transits from the initial state to the final state under certain constraints, with the interference from users. The test task can be understood as finding a path (practical and/or optimal) between the initial state and the final state, and this concerns the application of such algorithms as planning, decision, optimization, prediction, signal processing, system recognition, pattern recognition, etc.

\subsection{The Working Principles of VRVTT}

As has been discussed above, VRVTT is supposed to perform these basic functions: measurement, detection, test, simulation and training. Among these, simulation is the most important, since VRVTT has to go through a period of completely-virtual test, i.e., VR-based simulation of test processes and results, before it can be combined with real (physical) equipments to perform real tasks.

In the simulation function of VRVTT, prediction is very important, which includes applying the available test principles and experiences to the solution of real task. After the test scheme is decided and parameters properly selected, the aim of the simulation is to get a useful prediction of the performance of the real system, and display it in a virtual test environment.

The predictive test in VRVTT is based on the fact that many machine parts are made from the same type or similar type of materials, dimensions and shapes, the properties of the materials used are familiar to the manufacturers, and the effect of dimensions and shapes on the performance can be deduced from past test data.

The predictive test in VRVTT is to find and simulate the causal relation between the results and the type of materials, dimensions and shapes of the parts. Suppose the causal relation under consideration be expressed as:

$$
\mathrm{Y}(\mathrm{t})=\mathrm{F}(\mathrm{u}(\mathrm{t}), \mathrm{a}(\mathrm{t}))
$$

Where $\mathrm{t}$ is time, $\mathrm{Y}(\mathrm{t})$ represents test results or output, $\mathrm{u}(\mathrm{t})$ represents the variables which form the input or reason of the system, a(t) represents other unclear factors or noises which also affect the system, and $F()$ is the mathematical expression of the causal relation between the input and output of the system.

Since the input of the system can be simulated by the system by imitating physical systems or following relevant regulations, and noises can be added into the system without much difficulty, Eq. (2) will be useful in predicting the test process and results if the causal relation between the input and output of the system can be obtained. This relation can be obtained in many ways, and one effective method is through the technique of system recognition, which is based on the obtained data in the past, i.d., $\mathrm{Y}(\mathrm{t}), \mathrm{u}(\mathrm{t})(\mathrm{t}=1,2,3$,

VRVTT receives instructions from user through man-machine interface, and collects information of the test system, object and environment. Based on the a priori knowledge and algorithms in databases, VRVTT performs functions like control, prediction, collision detection, process optimization, simulation, decision-making, etc. As its output, VRVTT shows the simulation process and results in a virtual environment by means of measurement, detection and test process visualization, results visualization, results analysis, and process replay, etc. 


\section{Application of VR-Based Virtual Test Technology in Instrument Development}

\subsection{Application of VR-Based Virtual Test Technology in Instrument Development}

Nowadays developers of instruments are facing more and more pressures from the market, since the development cycle has to be shortened, and the flexibility of the whole development process needs to be increased. To meet such demands, virtual instruments (VI) have been adopted in many industries where instruments are needed [8][9]. The basic idea of VI is to replace the old-fashioned application-specific instruments with computer hardware and software, most typically by replacing the signal acquisition and processing units with computer algorithms, and replacing the old display panels and screens with computer display. In this way, a computer can be turned into a versatile data-processing instrument, which can serve more functions than the old application-specific instruments, thus reducing development cost drastically.

But VI has its disadvantages too. Besides the configuration of screens, buttons, knobs, switches and keypads on the front panel of the instrument, the users also want to know the complete appearance of the instrument in real applications, its working principles, signal flowcharts, wiring system, hardware configuration, display results, etc., preferably in visual and interactive forms, and this is exactly what VR-based virtual test technology is good at. So VR-based virtual test technology supplies a novel approach for instrument development, since the user can implore the instrument to be designed before it is made in a 3D virtual environment, and networked development can be easily implemented.

VRVTT can be very different for different applications, so here the case of nondestructive test (NDT) is taken as an example. Among various NDT methods, ultrasonic NDT has become the most important method adopted in many fields such as airplane, ship, automobile industries, medical science etc. for the detection of internal defects in metal and nonmetal parts[10]-[12]. This is due to the ultrasonic wave's special advantages of high sensitivity, good direction, strong penetration ability, and being harmless to human body. But in China, many manufacturers still use the traditional method of manual test, which requires the participation of professional personnel, and the efficiency and accuracy are rather limited.

VRVTT can do quite a lot in partly solving this problem. By setting up a model of the test system under design in advance in computer, the cost is greatly reduced, and flexibility is increased, since the model can be modified without causing much trouble. What's more, by performing virtual test and simulation in a computer-generated virtual environment, the professional personnel and skillful operators can both be trained in an effective way. Finally, since NDT industry has accumulated large quantities of test principles and experiences, which can be used to form knowledge bases and guidelines for the test tasks, intelligent prediction of test process and results are made not only possible but also practical. In fact, the state-of-the-art of ultrasonic NDT is to develop auto-detection systems and prediction models, and show test results in $3 \mathrm{D}$ rather than $2 \mathrm{D} .[13][14]$ 


\subsection{A Prototype VR-Based Instrument Development System}

A prototype virtual ultrasonic NDT system (VNDTS) is developed to show the application of VR-based virtual test technology in instrument development, and shows how a simple but helpful virtual test system can be implemented on a personal computer without much cost. Research work under study includes modeling of the NDT system, analysis of the NDT system, simulation of the NDT process, prediction and display of NDT results, and finally, the practical virtual test of real objects.

VNDTS adopts a client/server (C/S) configuration, and TCP/IP is used for communication protocol. VNDTS combines techniques such as Hypertext Markup Language (HTML), VRML, Servlet and Java to supply the system with various functions. On the server side, application programs include user management server, VRML world generating server and virtual testing server; client runs on webpages, which are downloaded with HTTP protocol, with Java Applet and VRML browser supplied. VRML supplies 3D viewing of the scenes, while Java Applet supplies user interface, deals with application protocol communication between client and server, communicates with VRML space through External Authoring Interface (EAI), downloads local models to client, and submits test results to server, etc. WWW server manages information on webpages, Java Applet, VRML files and virtual tests. Application server supplies services for clients through the webpages.

The handling of user's task can be on the server side or on the client side. If it is done on the server side, when the server accepts the requirement, a server application program will be run, and then the results will be transferred back to the client. The server is the core of the system, and supplies most of the functions, while the configuration of the client can be highly flexible. The $\mathrm{C} / \mathrm{S}$ structure facilitates the software system, simplifies the client software, and makes the use of VNDTS very convenient and easy.

Client can be a simple PC which has access to the Internet, while the server is the essential part of the system. For the sake of economy and convenience, VRML, HTML and JAVA are chosen for 3D modeling tool, webpage display and implementation of complex algorithms respectively.

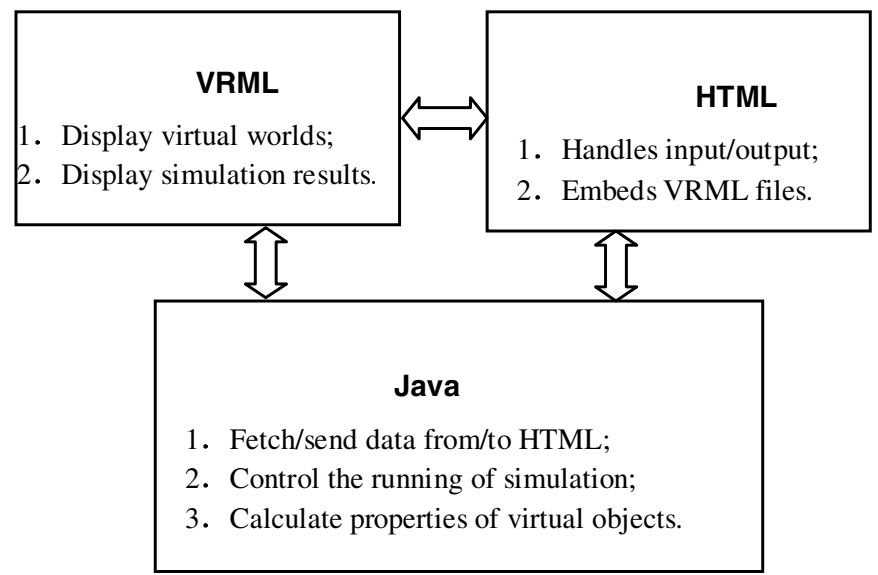

Fig. 3. Working principle of VNDTS 
The working principle of VNDTS is shown in Fig. 3. VRML and Java Applet are put into the same web page, and Java Applet serves as the core of simulation control. VRML does not have calculation function itself, and such functions are all implemented with Java. The 3D world browser used is CosmoPlayer.

When the system is started, it first enters a HTML mainpage to allow the user to select from several options. After the selection of system function and parameters, VNDTS allows the user to view $2 \mathrm{D}$ teaching materials or enters $3 \mathrm{D}$ display and interaction mode. VNDTS receives instructions and requirements from user through manmachine interface, and collects relevant information for the special task. After requirements are handled and calculations made, the results are sent back to the client.

Fig. 4 shows some pictures of VNDTS, which shows how a simple but helpful VRbased test system can be implemented on a personal computer without much cost. In Fig. 5, (a) shows a test table in a virtual test environment, and the user can interact with it by using the buttons supplied by CosmoPlayer. (b) shows a pseudo 3D display of NDT result, thus supplying more information than a 2D image. (c) is an image in $2 \mathrm{D}$, but color information is emphasized to help user locate the defects. (d) is the window where user can input the test data and parameters. Work under research is trying to combine the technique of scientific visualization with VR, so as to create 3D and vivid images of the tested part and internal defects.

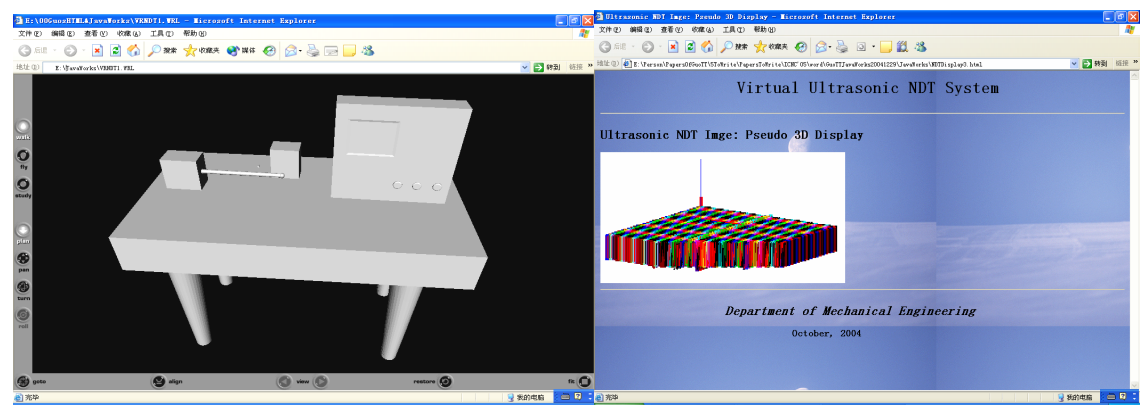

( a)

( b)

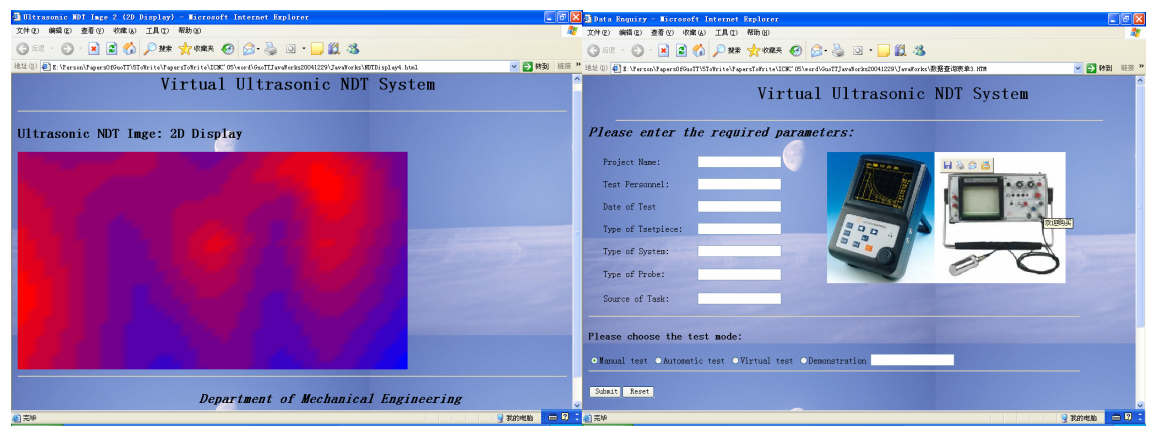

( c)

( d)

Fig. 4. Pictures of a virtual ultrasonic NDT system 
Main difficulties in developing VNDTS lie in modeling, including the geometrical, physical and movement modeling of both the virtual environment and test equipment and object. Users often ask for scenes as real as possible, and refreshing of scenes as fast as possible, while these contradictory requirements both mean huge demand of storage and calculation time. To ease this bottleneck, many ideas have been put forward, like LOD (level of detail) [15][16], in which scenes are represented in different levels of detail according to the direction and distance of the viewer, and image-based rendering[17][18], in which images are used directly to form a part of the virtual environment, especially when large area of background is concerned.

There are many kinds of data with different data format and time sequence flowing in VNDTS: data from VR peripheral equipments, data from computer vision module, data from database system, data created within VNDTS during the test and simulation process, data from actual test equipment and test object in the case of partially virtual VNDTS, etc., and some of them are contradicting to each other. All these must be coordinated and integrated into VNDTS. Technologies like data fusion, multi-sensor fusion, fuzzy pattern recognition and neural network can be used to solve this problem.

Though the system is just a prototype system with limited functions, the pictures show the potential of this approach in the field of test and simulation.

\section{Conclusions}

Test technology is facing more and more challenges, and simulation is proved to be an economic and useful tool in reducing cost and increasing reliability and products' quality. To supply a new approach for the solution of complex engineering test problems, this paper first gave a modified model of VR-based virtual test technology, and then discussed its application in instrument development. As a prototype example of VRVTT, this paper also introduced a VR-based virtual non-destructive-test system which is implemented with HTML, Virtual Reality Modeling Language (VRML) and Java.

Though for the moment the application of VRVTT is still immature and not very common, and the prototype system developed has just limited functions, but the effects are quite good, showing the potential of this approach in test and instrumentation, and it can be expected that with the development of test technology and computer hardware and software techniques, it'll become more and more practical to conduct test in virtual test environments, and the future of VRVTT is very promising.

\section{References}

1. Wilson, J.R., D'Cruz, M.: Virtual and interactive environments for work of the future. Int. J. Human-Computer Studies 64, 158-169 (2006)

2. Pouliquen, M., Bernard, A., Marsot, J., et al.: Virtual hands and virtual reality multimodal platform to design safer industrial systems. Computers in Industry 58, 46-56 (2007)

3. Pan, Z., Cheok, A.D., Yang, H., et al.: Virtual reality and mixed reality for virtual learning environments. Computers \& Graphics 30, 20-28 (2006)

4. Egerstedt, M., Hu, X., Stotsky, A.: Control of mobile platforms using a virtual vehicle approach. IEEE Transactions On. Automatic Control 46(11), 1777-1782 (2001) 
5. Tiantai GUO. Research on the Theory and Applications of VR-based Testing [PhD thesis]. Zhejiang University, Hangzhou, P. R. China. (August 2005) (In Chinese)

6. Guo, T., Zhou, X., Zhu, G.: Application Of CBR In VR-Based Test And SimilationSystem. In: Proc. of 2003 International Conference on Machine Learning and Cybernetics. Xian, China, 2-5 November 2003, pp. 2337-2340 (2003)

7. Burdea, G., Coiffet, P.: Virtual Reality Technology New York: A Wiley-Interscience Publication, John Wiley \& Sons, Inc., pp. 1-14 (1994)

8. Taner, A.H., Brignell, J.E.: Virtual instrumentation and intelligent sensors. Sensors and actuators 61, 427-430 (1997)

9. Hubert, C.G., McJames, S.W., Mecham, I., et al.: Digital imaging system and virtual instrument platform for measuring hydraulic conductivity of vascular endothelial monolayers. Microvascular Research 71, 135-140 (2006)

10. Ullah, F., Kaneko, S.: Using orientation codes for rotation-invariant template matching. Pattern Recognition 37(2), 201-209 (2004)

11. Yin, X., Morris, S.A., O’Brien Jr., W.D.: Experimental Spatial Sampling Study of the Real-Time Ultrasonic Pulse-Echo BAI-Mode Imaging Technique. IEEE Transaction on Ultrasonics and Frequency Control 50(4), 428-440 (2003)

12. Ma, H.W., Zhang, X.H., Wei, J.: Research on an ultrasonic NDT system for complex surface parts. Journal of Materials Processing Technology 12(9), 667-670 (2002)

13. Sadoun, B.: An efficient simulation scheme for testing materials in a nondestructive manner. Information Sciences 137, 43-51 (2001)

14. Fenster, A., Surry, K., Smith, W., et al.: 3D ultrasound imaging: applications in imageguided therapy and biopsy. Computers \& Graphics 26, 557-568 (2002)

15. Jeong-Hwan, A., Dong-Keun, L., Ho, Y.-S.: Compact representation of 3D models using mesh merging[C]. TENCON '97. IEEE Region 10 Annual Conference. Speech and Image Technologies for Computing and Telecommunications Proceedings of IEEE, 1, 323-326 (1997)

16. Gobbetti, E., Bouvier, E.: Time-critical multiresolution rendering of large complex models[J]. Computer-Aided Design 32(13), 785-803 (2000)

17. Mukaigawa, Y., Mihashi, S., Shakunaga, T.: Photometric image-based rendering for virtual lighting image synthesis[C]. In: Augmented Reality 1997 (IWAR '99) In: Proceedings. 2nd IEEE and ACM International Workshop on, 1999, pp. 115-124 (1999)

18. Hansard, M.E., Buxton, B.E.: Image-based rendering via the standard graphics pipeline[C]. Multimedia and Expo 2000, ICME 2000. In: 2000 IEEE International Conference on. 2000, vol. 3, pp. 1437-1440 (2000) 